\title{
Mathematical model for decision-making neural circuits controlling food intake
}

\author{
G. M. BARNWELL \\ Bioengineering Department, South west Research Institute, San Antonio, Texas 78284 \\ and \\ F. S. STAFFORD \\ Bioengineering Department, University of Texas Health Science Center, San Antonio, Texas 78284
}

\begin{abstract}
A mathematical model of two decision-making neural circuits for controlling food intake is presented. Results of some initial analog computer simulations are presented and demonstrate that the model exhibits stable steady states and thresholds for steady state transitions corresponding to experimentally observed behaviors characteristic of lateral and ventromedial hypothalamic interactions in food intake control. The relations of the model and simulations to experimental evidence are discussed.
\end{abstract}

One of us recently proposed a theory of mammalian food intake control and a mathematical model of two mutually inhibitory decision-making neural circuits for controlling food intake (Barnwell, 1974). The decision-making model shows how closed-loop mutually inhibitory positive feedback neural circuits can monitor metabolic states, integrate exteroceptive and interoceptive sensory inputs, and make decisions via steady state transitions that commit the animal to a given behavioral mode, i.e., the model shows how the animal decides when to eat and when to stop. The model is described below and results of initial analog computer simulations are presented and related to experimental evidence.

\section{DECISION-MAKING MODEL}

It has been suggested that positive feedback processes are involved in motivational arousal circuits that initiate feeding or in stimulus properties that maintain and facilitate feeding (DeRuiter, 1967; Fisher, 1969; Hoebel, 1969: LeMagnen, 1969; Wiepkema, 1971), and that closed-loop reverberatory circuits of neurons selectively activated by different chemicals or transmitters could play a fundamental role in the biological basis of drive and motivation (Fisher, 1964, 1969; Grossman, 1968; Morgane, 1969; Stein, 1968). The positive feedback circuits indicated by the evidence involve portions of the medial forebrain bundle, lateral hypothalamus (LH), and ventromedial hypothalamus (VMH), reticular system, limbic system, and other regions. There is substantial evidence for mutually inhibitory interactions of $\mathrm{LH}$ and VMH (Anand, Subberwal, Manchanda, \& Singh, 1961; Anand, Chhina, Sharma, Duas, \& Singh, 1964; Oomura, Kimura, Ooyama, Maeno, Iki, \& Kunyoshi, 1964; Oomura, Ooyama, Yamamoto, Ono, \& Kobayashi, 1969;

J. D. Davis sponsors and takes full editorial responsibility for this paper.
Oomura, Ooyama, Naka, Yamamoto, Ono, \& Kobayashi, 1969). Anatomical evidence also indicates reciprocal relations between LH and VMH (Arees \& Mayer, 1967; Millhouse, 1973a, b).

The model below assumes that feeding and satiety decisions result from the interactions of a feeding circuit and a satiety circuit, each consisting of hypothalamic and extrahypothalamic neurons. Each circuit is assumed to consist of a large number of mutually excitatory neurons in a reverberatory circuit or positive feedback loop configuration, such that only excitatory synapses are formed between pairs of neurons in the circuit. Reverberatory activity is assumed to occur in both circuits. No implication of homogeneity of LH or VMH is intended. Other types of neurons probably exist in the $\mathrm{LH}, \mathrm{VMH}$, or other regions through which the feeding and satiety circuits course. The present model includes two types of neurons. However, effects of interneurons or other types of neural circuits, e.g., for water intake, can easily be incorporated into the model, and one of the potential uses of such models is to make theoretical predictions of the behavior of complex multicircuit systems.

The activity, $\mathrm{N}$, in a neural circuit is defined as the total number of neural firings occurring per second. A particular activity level could result from a few neurons firing at high rates, many neurons firing at low rates, or intermediate combinations. Properties of component neurons and their interconnections in a circuit are assumed to be similar, and certain of these properties (e.g., degree of convergence and divergence, membrane time constant, refractory period, axonal transmission time) determine a saturation level or maximal activity level, $\eta$, for the circuit. Since the total activity can never exceed $\eta$, and the activity is represented by $\mathrm{N}$, the degree of inactivity is defined by $(\eta-\mathrm{N})$.

The rate of increase of activity in a positive feedback neural circuit is proportional to the product of the 


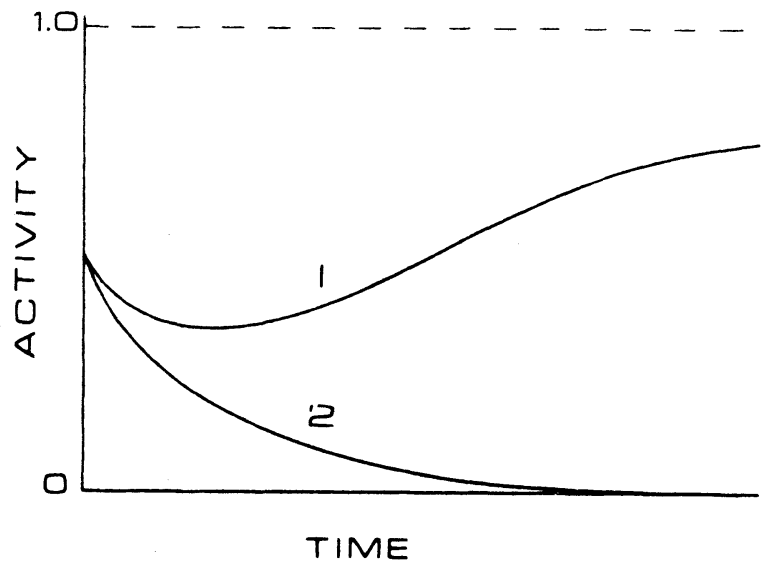

Figure 1. With same initial activity levels, activity in circuit receiving stronger excitation increases, while activity in opposing circuit is suppressed. Simulation time span in figures is 7.5 sec. Numbers on curves correspond to circuits.

present activity times the degree of inactivity times the sum of the internal connectivity (including the positive feedback effect) and the amount of external excitation $\mathrm{E}$ arriving, i.e., the rate of increase is proportional to $\mathrm{N}(\eta-\mathrm{N})(\gamma+\beta \mathrm{E})$, where $\gamma$ is the internal connectivity due to the positive feedback loop connective pattern, while $\beta$ is a lumped term including both the average weighted effects of chemical inputs or incoming excitatory fibers and the average connectivity among neurons in a neural mass or network not arranged in a positive feedback loop. The above expression is a form of the logistic equation in population biology, whose saturation behavior is characterized by the well-known sigmoid curve. Thus, the rate of increase is small for very small values of $\mathrm{N}$ or $(\eta-\mathrm{N})$, and maximal at $\mathrm{N}=\eta / 2$. The saturation property results in a stable positive feedback system.

The rate of decrease of activity is proportional to the degree of external inhibition of the active subpopulation of neurons and to a proportional decay rate $\mathrm{LN}$, which is the rate at which active neurons become inactive in the absence of other influences.

Since all the above activity changes are proportional to present activity, $\mathrm{N}$ is a multiplier for each term, and relative activity is obtained by the ratio $\mathrm{N} / \eta$.

The general representation of the two-circuit model is given in Equations (1) and (2),

$$
\frac{d \mathrm{~N}_{1}}{d \mathrm{t}}=\frac{\mathrm{N}_{1}}{\eta_{1}}\left[\left(\eta_{1}-\mathrm{N}_{1}\right)\left(\gamma_{1}+\sum_{\mathbf{j}} \beta_{1 \mathbf{j}} \mathrm{E}_{1} \mathbf{j}\right)-\alpha_{1} \mathrm{~N}_{2}-\underset{\mathbf{K}}{\sum} \delta_{1} \mathbf{K}_{1} \mathbf{K}-\mathrm{L}_{1}\right]
$$

$$
\frac{d \mathrm{~N}_{2}}{d \mathrm{t}}=\frac{\mathrm{N}_{2}}{\eta_{2}}\left[\left(\eta_{2}-\mathrm{N}_{2}\right)\left(\gamma_{2}+\sum_{\mathbf{j}}{\beta_{2} \mathrm{j}}_{2 j}\right)-\alpha_{2} \mathrm{~N}_{1}-\sum_{\mathbf{K}} \delta_{2} \mathrm{~K}^{\mathrm{I}_{2} \mathrm{~K}}-\mathrm{L}_{2}\right]
$$

where $N_{1}$ and $N_{2}$ are, respectively, the activity levels in Circuits 1 (feeding) and 2 (satiety) and $\eta_{1}$ and $\eta_{2}$ are the saturation numbers denoting the maximum possible level of activity in the respective circuits. Since activity is a multiplier in both equations, $\mathrm{N}_{1}$ and $\mathrm{N}_{2}$ are assumed to always be positive valued; this mathematical restriction agrees with one's biological intuition that activity in a neural circuit could be arbitrarily small but never zero. The internal connective strengths due to the positive feedback effects are denoted by $\gamma_{1}$ and $\gamma_{2}$. The effects of all excitatory chemical and neural inputs are represented by the sums of terms involving $\beta_{1 j} E_{1 j}$ and $\beta_{2 j} E_{2 j}$, where $j$ is the index of summation. For example, excitatory effects of glucose on VMH neurons could be incorporated by the term $\beta_{21} E_{21}$, while excitatory effects of stomach distention on VMH neurons could be represented by $\beta_{22} E_{22}$. The terms $\beta_{1 j}$ and $\beta_{2 j}$ are lumped parameters as described above, while $E_{1 j}$ and $E_{2 j}$ represent the magnitudes of the excitatory inputs. The effects of all inhibitory chemical and neural inputs are represented by the sums involving $\delta_{1 \mathrm{~K}} \mathrm{I}_{\mathrm{K}}$ and $\delta_{2 \mathrm{~K}} \mathrm{I}_{2 \mathrm{~K}}$, where $\delta_{1 \mathrm{~K}}$ and $\delta_{2 \mathrm{~K}}$ denote connective strengths of inhibitory inputs and $\mathrm{I}_{1 \mathrm{~K}}$ and $\mathrm{I}_{2 \mathrm{~K}}$ denote inhibitory input magnitudes. The inhibitory connective strength of Circuit 2 on Circuit 1 is denoted by $\alpha_{1}$, and that of Circuit 1 on Circuit 2 by $\alpha_{2}$, while $L_{1}$ and $L_{2}$ are proportional decay factors.

\section{SIMULATION RESULTS}

The simulations and figures were produced, respectively, with an EAI 580 analog computer and EAI 1125 Variplotter. An extensive preliminary simulation study designed to examine the qualitative behavior of the model has established that the behavior corresponds to previous theoretical predictions (Barnwell, 1974) and to several experimental observations. Since the purpose of this preliminary study was to determine whether the model would exhibit behaviors qualitatively corresponding to certain experimental observations of LH-VMH interactions, the levels of excitation and inhibition and the parameter values are regarded as relative strengths of dimensionless numbers ranging from 0.0 to 1.0 .

The first question examined was whether an imbalance of excitation would produce a maximal activity level in the circuit receiving the excitation and minimal activity level in the opposing circuit. For equal numbers of neurons, equal inhibition, and loss in both circuits, activity in the circuit receiving a greater amount of excitation increased to the maximal level, while activity in the second circuit was depressed. An example of these simulations is shown in Figure 1, where Curve 1 represents activity in Circuit 1 , and Curve 2 represents activity in Circuit 2. Values for this simulation were $\eta_{1}=\eta_{2}=1.0, \mathrm{~N}_{1}(0)=\mathrm{N}_{2}(0)=.5, \gamma_{1}=\gamma_{2}=.1$, $\mathrm{L}_{1}=\mathrm{L}_{2}=.1, \mathrm{I}_{1}=\mathrm{I}_{2}=0, \alpha_{1}=\alpha_{2}=.5, \beta_{1} \mathrm{E}_{1}=.125$, and $\beta_{2} E_{2}=0$.

With both circuits having equal initial activity levels, excitatory inputs to Circuit 1 increased activity of this 
circuit and decreased that of Circuit 2, both eventually approaching respective maximal and minimal saturation values. The initial decrease in activity of both circuits is due to the relatively strong reciprocal inhibition.

A larger excitatory input to Circuit 1 produced a more rapid attainment of saturation levels, as shown in Figure 2. Values for this simulation were the same as for Figure 1, except that $\beta_{1} E_{1}=.250$.

Although a moderate imbalance of excitation could drive activity in one circuit to its maximal value beginning at intermediate activity levels, very strong amounts of excitation or inhibition were necessary for reversing saturation level dominance of one circuit over the other. With initial conditions of .99 for relative activity in Circuit 1 and .01 for relative activity in Circuit 2, and all other initial values identical to those used for Figure 2, there was only a slight change in both transient and steady state activity levels. Increasing $\beta_{2} E_{2}$ from 0 to .1 , a value equal to $\beta_{1} E_{1}$, produced no change in this behavior. Similarly, values of $\beta_{2} E_{2}$ up to .4 produced no effect. However, at $\beta_{2} E_{2}=.43$, the dominance of Circuit 1 over Circuit 2 began to be reversed by the much stronger input excitation level in Circuit 2, and a complete reversal was attained at $\beta_{2} E_{2}=.6$. Thus, thresholds for reversals exist. Figure 3 shows an example for which $\beta_{2} E_{2}=.75$.

\section{DISCUSSION}

The preliminary simulations have shown that the model exhibits predicted qualitative behavior corresponding to a variety of experimental observations on LH-VMH interactions mentioned below. Beginning at intermediate levels of activity in the two circuits, only a moderate imbalance of excitation/inhibition drives the circuit with most excitation toward its saturation activity level. This corresponds to any situation in which increasing the value of any variable or combination of variables that excites Circuit 1 or inhibits Circuit 2 will

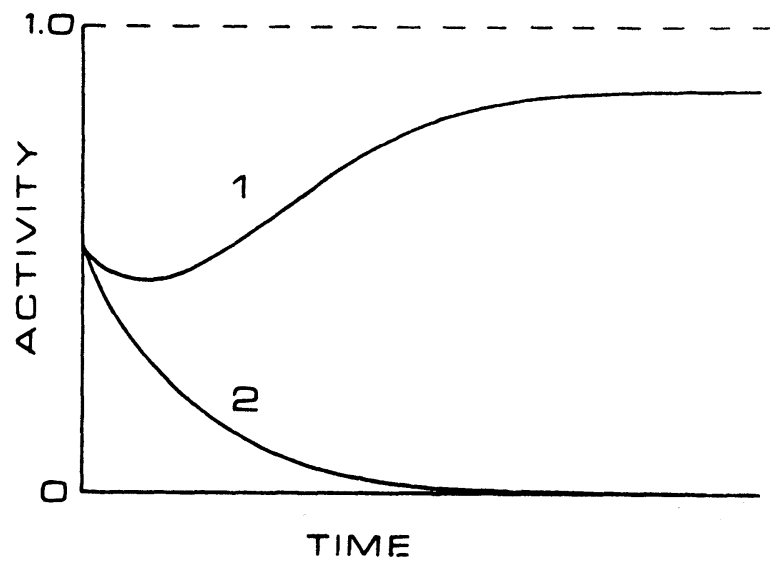

Figure 2. Langer imbalance of excitatory inputs results in more rapid attainment of steady state levels.

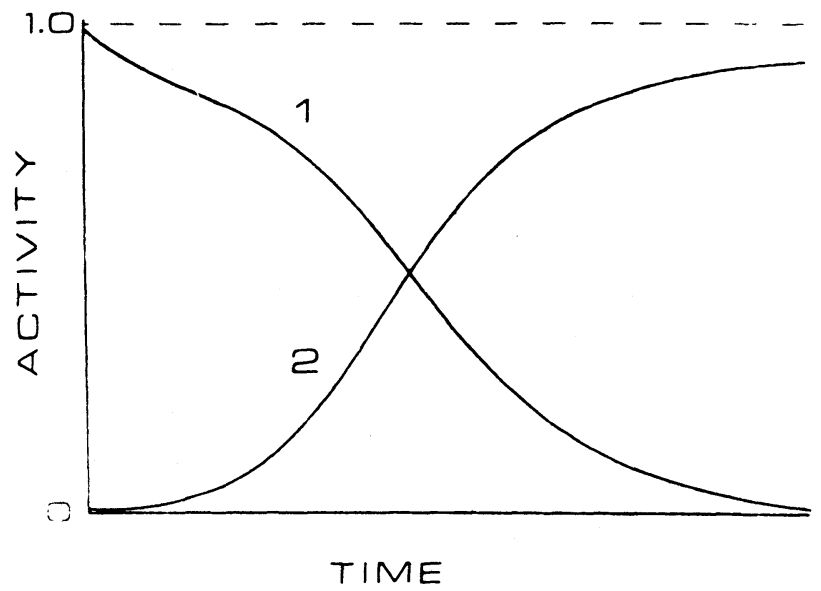

Figure 3. Very strong excitation of suppressed circuit or inhibition of dominant circuit can reverse relative activity levels.

lead to increased activation and dominance of Circuit 1 over Circuit 2, and hence, result in the characteristic behavior; similarly, activity in Circuit 2 could become stronger than Circuit 1 by a reversed effect. Such behavior is an analog for a moderately food-deprived animal that is exposed to food-associated stimuli and then begins to eat.

When activity in one circuit becomes completely dominant to the other circuit, very strong excitation of the suppressed circuit or very strong inhibition of the dominant circuit is necessary to reverse the situation and change the behavior as illustrated by Figure 3, e.g., electrical stimulation of the $\mathrm{LH}$ can produce feeding in a satiated animal. Rebound effects were also demonstrated; such effects are a consequence of the stable steady states in the model.

Thresholds were shown to exist for steady state transitions involving reversals of circuit dominance. The thresholds are significant system properties since they are variable, they guarantee that ongoing behavior will not be disrupted by small fluctuations (e.g., a satiated animal will not begin to eat in response to the sight of food), and any combination of variable values that exceeds the existing threshold will reverse the dominance and result in the opposite behavioral mode. This latter property of thresholds corresponds to the suggestion that an optimization process controls decision making, in that the neural circuits are responding to a weighted sum of current values of all variables affecting food intake (Barnwell, 1974). Experimental modifications such as vagotomy or feeding with a gastric fistula correspond to eliminating one or more of the inputs in the summation so that higher values of the remaining variables are necessary to achieve the extant threshold level.

In summary, a brief behavioral interpretation is that if there is a high activity level in the feeding circuit and food-associated stimuli are present, feeding will occur. 
Feeding is terminated when satiety circuit activity is significantly increased by appropriate feedbacks such as increased blood glucose, temperature, or stomach distension. Feeding initiation and termination correspond to steady state shifts in the model. A further paper will show how high feeding circuit activity and presence of food-associated stimuli could control feeding reflexes to initiate and maintain feeding.

Experimental validation of the model would be based on electrophysiological recording and statistical sampling techniques similar to those of Oomura, Ooyama, Yamamoto, Ono, \& Kobayashi (1969) and Oomura, Ooyama, Naka, Yamamoto, Ono, and Kobayashi (1969). Activity levels would be estimated from the average firing frequency of sampled neurons, with sampling regions distributed throughout the circuit. These problems will be treated in detail in subsequent papers that will include specific simulations of previous experimental results and predictions based on measurable physiological/behavioral parameters. The advantages of the model are that it exhibits the nonlinearities and threshold phenomena common to biological systems, it integrates a wide variety of variables known to affect food intake, it is directly relatable to specific neurochemical pathways, and it is capable of simulating a wide variety of experiments and; making predictions.

\section{REFERENCES}

Anand, B. K., Subberwal, U., Manchanda, S. K., \& Singh, B. Glucoreceptor mechanism in the hypothalamic feeding centres. Indian Journal of Medical Research, 1961, 49, 717-724.

Anand, B. K., Chhina, G. S., Sharma, K. N., Dua, S., \& Singh, B. Activity of single neurons in the hypothalamic feeding centers: Effect of glucose. American Journal of Physiology, 1964, 207, $1146-1154$.

Arees, E. A., \& Mayer, J. Anatomical connections between inedial and lateral regions of the hypothalamus concerned with food intake. Science, 1967, 157, 1574-1575.
Barnwell, G. M. Mammalian food intake control: A theory and mathematical model. V International Conference on Physiology of Food and Fluid Intake, Jerusalem, Israel. October 10-13, 1974.

DeRuiter, L. Feeding behavior of vertebrates in the natural environment. In: C. F. Code (Ed.), Handbook of physiology (Section 6). Alimentary canal (Vol. 1). Food and water intake. Washington, D. C: American Physiological Society, 1967. Pp. 97-116.

Fisher, A. E. Chemical stimulation of the brain. Scientific American, 1964, 210,60-68.

Fisher, A. E. The role of limbic structures in the central regulation of feeding and drinking behavior. Annals of the New Y ork Academy of Science, 1969, 157, 894-901.

Grossman, S. P. Hypothalamic and limbic influences on food intake. Federation Proceedings, 1968, 27, 1349-1360.

Hoebel, B. G. Feeding and self-stimulation. Annals of the New York Academ y of Science, 1969, 157, 758-778.

LeMagnen, J. Peripheral and systemic actions of food in the caloric regulation of intake. Annals of the New York Academy of Science, 1969, 157, 1126-1157.

Millhouse, $O$. E. The organization of the ventromedial hy pothalamic nucleus. Brain Research, 1973, 55, 71-87.

Millhouse, $O$. E. Certain ventromedial hypothalamic afferents. Brain Research, 1973, 55, 89-105.

Morgane, P. J. The function of the limbic and rhinic forebrain-limbic midbrain systems and reticular formation in the regulation of food and water intake. Annals of the New York Academy of Science, 1969, 157, 806-848.

Oomura, Y., Kimura, K., Ooyama, H., Maeno, H., Iki, M., \& Kuniyosh $\ddot{i}, M$. Reciprocal activities of the ventromedial and lateral hypothalamic areas of cats. Science, 1964, 143, 484-485.

Oomura, Y., Ooy ama, H., Yamamoto, T., Ono, T., \& Kobayashi, $N$. Behavior of hypothalamic unit activity during electrophoretic application of drugs. Annals of the New York Academy of Science, $1969,157,642-665$. (a)

Academy of Science, 1969,157, Naka, F., Yamamoto, T., Ono, T., \& Kobay ashi, N. Some stochastical patterns of single unit discharges in the cat hypothalamus under chronic conditions. Annals of the New York Academy of Science, 1969, 157, 666-689. (b)

Stein, L. Chemistry of reward and punishment. In: D. H. Efron (Ed.). Psychopharmacology, a review of progress: 1957-1967, Washington, D. C: U. S. Government Printing Office, 1968. Pp. 105-123.

Wiepkema, P. R. Positive feedbacks at work during feeding. Behaviour, 1971, 39, 266-273.

(Received for publication March 14, 1975.) 